\title{
Asset Allocation with Markovian Regime Switching: Efficient Frontier and Tangent Portfolio with Regime Switching*
}

\author{
André Barbosa Oliveira ${ }^{* *}$ \\ Pedro L. Valls Pereira ${ }^{* * *}$
}

\begin{abstract}
Asset allocation is important for diversifying risk and realizing gains in the financial market. It involves decisions taken under uncertainty based on statistical methods. Returns on financial assets generally present regime switching and there are different distributions of returns in bull and bear markets. Regime switching in the data generating process for returns makes it necessary to reformulate the asset allocation problem. This paper develops asset allocation models with regime switching. Due to the comparative study of asset allocation, portfolios with regime switching enable the space of risk and return to be increased, reduce the risk for each level of return at the mean variance efficient frontier, and have the best risk-return relationship over time.
\end{abstract}

Keywords: Portfolio theory; Time series models with Markovian regime switching; Optimization theory.

JEL codes: G11; C34; C61.

Submitted on $17 / 02 / 2017$. Revised on $18 / 10 / 2017$.

We are grateful for the comments of an anonymous reviewer, Marcelo Fernandes, Emerson Marçal, Marcos Eugênio da Silva, and Flávio Augusto Ziegelmann. Any errors are the authors' responsibility. The second author is grateful for the funding from $\mathrm{CNPq}(309158 / 2016-8)$ and from FAPESP (2013-22930-0)

** Department of Economics - UFF and CEQEF-FGV. Email: aboliveira@id.uff.br.

*** São Paulo School of Economics - FGV and CEQEF-FGV. Email: pedro.valls@fgv.br 


\section{Introduction}

The financial markets are one of the most important markets in the economy. They enable agents to transfer wealth between different time periods and states of nature, as well as sharing and diversifying risks (Mas-Colell et al. (1996)). Financial innovations increase the possibilities for transactions and efficiencies in an economy under uncertain (Cochrane (2005)). Agents operate in the financial market by offering different financial products to buyers, who have different attitudes in relation to risk and trade assets in the search for returns.

Asset portfolio composition is one of the main practices in the financial market. The most popular approach to asset allocation is that of Markowitz (1952), who states that agents should diversify their investments in order to minimize risks. Investors want to invest in a variety of assets that have diversified risks and more attractive returns compared to investing in a smaller number of assets. Even outside the financial sector, commercial companies and manufacturers develop strategies based on asset allocation principles in order obtain more advantageous client and supplier portfolios.

Asset allocation consists of the distribution of agents' wealth between a set of assets. Portfolio allocation depends on agents' preferences in terms of expected risks and returns, where the estimation of risks and returns is an important stage in asset allocation analysis. The allocations depend on the conditional expectations for the risks and returns of the assets. Asset allocation is a sequential process that depends on estimations of the conditional distribution of returns.

Returns on financial assets alter over time. In certain periods under a state of normality markets are calm and returns change slowly with low variability; in other periods when there is a state of crisis markets become agitated with the arrival of new information and returns behave with less predictability and more volatility. Various empirical studies apply regime switching models with a data generating process that is conditional on state (Hamilton (1989) and Hamilton (1994)) to describe the returns and volatility of financial assets (Franses and van Dijk (2005); Hamilton and Susmel (1994)).

The mean variance frontier is the set of risks and returns of financial assets and the efficient frontier lies over this set, establishing minimum risk allocations for 
a given average portfolio return. The mean variance frontier defines an asset set chosen by agents whose risk and return relationship depends on their preferences in the face of uncertainty. For an economy with different possible states of nature the agent chooses a portfolio plan that is contingent on the states of nature.

The mean variance approach to risk diversification is widely applied in finance. However, the optimal portfolios established by the mean variance frontier, such as the tangent portfolio, tend to present low returns performance (Jagannathan and Ma (2003); DeMiguel et al. (2009)). Due to the optimality condition of the mean variance portfolio, its portfolios tend to be concentrated in few assets and its allocations are concentrated in assets with higher returns and lower covariances, which are the estimates that are most sensitive to external observations and estimation sampling errors (Fusai and Roncoroni (2008)).

Mean variance portfolios define optimal diversification choices supposing that we have the populational risk and return parameters; however, the optimization is based on moment estimates that have sampling errors and differ from actual risks and returns (Michaud (1989); Scherer (2002)). Mean variance portfolios do not take into account the different states of nature that make the prediction errors in the unconditional moment estimates greater. The joint distribution of returns is different in bull and bear markets. Optimal risk diversification should consider that risks and returns depend on states of nature, the joint distribution of the returns are not i.i.d., as well as an invariant of the regimes over time, so that agents need to evaluate the probabilities of the states of nature to make their asset allocations.

For this reason, this article proposes studying the composition of optimal portfolios with regime switching. The risk and return for the allocations expected at the efficient frontier with regime switching would be equal to those of the portfolios at the efficient frontier without regime switching, and for the same average return they would have an equal expected risk. However, efficient frontier allocations with regime switching are the correct specification when the joint distribution of returns has regime switching. In cases with regime switching, the efficient frontier allocations that are invariant to the states do not correspond to optimal diversification, since besides the risks and returns being inadequate estimates, the problem would present a specification error. In this paper, we re-specify the asset allocation problem 
by developing the set of asset choices and optimal portfolios when the environment has distinct states, with risk and return being conditional on the states. The allocation with regime switching is more diversified that the mean variance portfolios and the optimal portfolios with regime switching introduced in this study have a better risk-return relationship than the traditional mean variance portfolios.

This article is organized in the following way: after this introduction, in the second section we characterize asset allocation with an intertemporal choice under uncertainty that is dependent on the states, using a current information set, where this information set and the estimates adapt over time. The third section addresses the statistical model for estimating the risks and returns for the allocation with uncertainty dependent on the states of nature, presenting the time series models with Markovian regime switching. The fourth section establishes the mean variance frontier with regime switching, the whole set of expected risks and returns in which the investors make their portfolio choices in the states. The fifth section empirically applies the portfolio with regime switching, comparing the traditional portfolios ${ }^{1}$ for the most liquid risk assets in the Brazilian stock market. The sixth section contains the final remarks.

\section{Conditional Asset Allocation}

Asset allocation is a choice made under uncertainty, where in the current period agents choose which assets they will invest in with risks and returns that will be realized in the next period. The uncertainty in the asset market implies that the return on a portfolio is unknown, with the gains on the asset allocation being uncertain and dependent on the states. Returns are different in bull and bear markets. We represent the uncertainty in asset allocation by states of nature. The return on the asset allocation depends on the random result of the returns over the state of nature in which the return is found.

States of nature are a description of the possible results of uncertainty, which will be denoted for one state by $s$ among $S$ possible states of nature for the set of states $s \in\{1,2, \ldots, S\}$.

\footnotetext{
1 The description of the models for the comparisons is presented in appendix.
} 
The asset market will have $N$ risk assets, which can be represented by the vector $R(s)=\left[R^{1}(s), R^{2}(s), \cdots, R^{N}(s)\right]^{\prime}$, where $R^{i}(s)$ represents the return on the $i-t h$ asset in state $s$.

The asset allocation problem consists of defining how much to invest in each stock or security in the market, establishing a portfolio represented by the vector $\omega=$ $\left[\omega_{1}, \omega_{2}, \cdots, \omega_{N}\right]^{\prime}$ where $\omega_{i} i \in\{1, \cdots, N\}$ represents the fraction of income invested in each asset. The return on a portfolio is a random variable, since the allocations are defined before the uncertainty comes to pass. Agents form expectations about the expected risks and returns for the states of nature in order to make allocations. The expectations regarding risks and returns for the future are what will guide the allocations. Gains from asset allocations are associated with an accurate prediction of market risks and future returns and expectations about the state of the economy.

Traditionally, expected return is represented by mean return and risk is represented by the variance in returns, that is, the spread of the distribution of returns around the mean.

When the asset allocation is at the mean variance frontier, agents choose the allocations that minimize the risk of their portfolio for a given return, or the dual problem of maximizing the return on their portfolio for a given risk. Thus, in the allocation at the mean variance frontier problem agents make their allocation in the current period, $t$, for the next period, $\omega_{t+1}$, which minimizes the risks given an expected return on the portfolio that is conditional on the current information set, $I_{t}$. The asset allocation problem at the conditional mean variance frontier is given by:

$$
\begin{array}{ll}
\min _{\left\{\omega_{t+1}\right\}} & \omega_{t+1}^{\prime} \operatorname{Var}\left[R_{t+1}(s) \mid I_{t}\right] \omega_{t+1} \\
\text { s.t. } & \omega_{t+1}^{\prime} E\left[R_{t+1}(s) \mid I_{t}\right]=\mu \\
& \omega_{t+1}^{\prime} \iota=1
\end{array}
$$

where $\omega_{t+1}$ is the vector of weights of the portfolio of dimension $N \times 1 ; \mu$ is the expected return on the portfolio and $\iota$ is a unitary vector of dimension $N \times 1$.

The conditional asset allocation approach emphasizes the sequential and adaptive aspect of portfolio management, where agents alter their allocations over time 
as the risk and returns estimates are updated with the arrival of new information and assessments regarding the state of nature.

The agents define a portfolio $\omega(s)$ for each state, $s \in S$. If the state is $s=1$, the optimal allocation is $\omega(1)$; on the other hand, if the state is $s=2$, the portfolio is $\omega(2)$. For any state $s \in S$. the optimal allocation for the investor is $\omega(s)$. However, the investors do not know in a particular period which state of nature will occur in the next period and choose the optimal allocation in the current period, in an environment with uncertainty associated with the states, using the expected allocation conditional on the states expressed as the sum of the allocation of each state weighted by the probability of the state.

For any sub-optimal allocation $\widetilde{\omega}$, the agent incurs the loss $L(\widetilde{\omega})=(\widetilde{\omega}-\omega(s))^{2}$. In an economy with uncertainty dependent on states, for the next period agents choose the allocation that minimizes the expected quadratic loss conditional on the current information $\min _{\{\omega\}} E\left[(\widetilde{\omega}-\omega(s))^{2} \mid I_{t}\right]$, establishing the optimal allocation using the expected portfolio conditional on the states $\omega_{t+1}^{*}=\omega_{t+1}(1) P\left\{S_{t+1}=\right.$ $\left.1 \mid I_{t}\right\}+\cdots+\omega_{t+1}(s) P\left\{S_{t+1}=s \mid I_{t}\right\}$.

\section{Time Series Model with Regime Switching}

The time series model with regime switching enables dependent and non-identically distributed time series to be described (Krolzig (1997)). The time series model with regime switching is a dynamic model, where the parameters of the data generating mechanism depend on the states over time.

The joint distribution of the time series vector $y_{t}=\left[y_{1, t}, y_{2, t}, \cdots, y_{N, t}\right]^{\prime}$ is multivariate normal. Assuming that we only have two regimes $S \in\{1,2\}, M S(2) M V N D$ (Markov Switch Multivariate Normal Distribution in the notation of Krolzig (1997)), the Data Generating Process (D.G.P.) is given by:

$$
y_{t}= \begin{cases}E\left(S_{t}=1\right)+\varepsilon_{t, S_{t}=1} & \text { if } S_{t}=1 \\ E\left(S_{t}=2\right)+\varepsilon_{t, S_{t}=2} & \text { if } S_{t}=2\end{cases}
$$


where $\varepsilon_{t, S_{t}=1} \sim N\left(0, \Omega\left(S_{t}=1\right)\right), \varepsilon_{t, S_{t}=2} \sim N\left(0, \Omega\left(S_{t}=2\right)\right), P=\left[\begin{array}{ll}p_{11} & p_{12} \\ p_{21} & p_{22}\end{array}\right]$ ,$s \in\{1,2\}$ and $E\left(S_{t}=s\right)$ is the mean vector in regime $s, \Omega\left(S_{t}=s\right)$ is the covariance variance matrix in regime $s$, and $p_{i j}$ are the probabilities of transition.

These probabilities of transition are described by the Markov chain and the property of the transition between the states over the course of the realizations of the stochastic process only depends on the present state and on the state to be assumed in the next period, and it is independent of the past states (Ross (2007)), i.e.:

$$
P\left\{S_{t}=j \mid S_{t-1}=i, S_{t-2}=i_{n-2}, \cdots, S_{0}=i_{0}\right\}=P\left\{S_{t}=j \mid S_{t-1}=i\right\}=p_{j i}
$$

These probabilities of transition $p_{j i}=P\left\{S_{t}=j \mid S_{t-1}=i\right\}$ describe the probabilities of transition from regime $i$ in period $t-1$ to regime $j$ in period $t$, which are considered constant in time in the Markov chain.

When the conditional means have no structure we have the mixture of normal distributions model. In other situations we can use an autoregressives model to model the conditional mean. In the latter case we can have changes in the mean, in the intercept, in the autoregressive coefficients, or in the variances. To estimate these models with regime switching we use an iterative procedure, applying the Expectation Maximization algorithm (Hamilton (1994); Kim and Nelson (1999)).

In the time series models with regime switching, agents make predictions by assessing the distribution of each state and the probabilities of the regimes. For example, the predictions for the mean and variance in the next period are given by:

$$
\begin{aligned}
E\left[y_{t+1} \mid I_{t}\right] & =E\left(S_{t}=1\right) \times P\left(S_{t+1}=1 \mid I_{t}\right)+E\left(S_{t}=2\right) \times P\left(S_{t+1}=2 \mid I_{t}\right) \\
\operatorname{Var}\left[y_{t+1} \mid I_{t}\right] & =\Omega\left(S_{t}=1\right) \times P\left(S_{t+1}=1 \mid I_{t}\right)+\Omega\left(S_{t}=2\right) \times P\left(S_{t+1}=2 \mid I_{t}\right)
\end{aligned}
$$

\section{Characterization of the Efficient Frontier with Regime Switching}

This section presents the problem of the efficient frontier with regime switching, establishing the set of returns and risks for the efficient allocations when the joint distribution of returns has regime switching. 
In an economy with $s$ states of nature or possible regimes that can occur in the next period $(t+1)$ based on an initial moment $(t), s \in\{1,2, \ldots, S\}$, $s \in S$, for example. Suppose that the economy has $N$ assets with a returns vector $R(s)=\left[R^{1}(s), R^{2}(s), \cdots, R^{N}(s)\right]^{\prime}$; where the expected returns vector is $E(s)=\left[\mu^{1}(s), \mu^{2}(s), \cdots, \mu^{N}(s)\right]^{\prime}$ if state of nature $s$ occurs in the next period and the covariance variance matrix of the returns on the assets will be denoted by $\Omega(s)$, $s \in S$.

In the analytical derivation of the mean variance frontier with regime switching, we consider the hypotheses of free portfolio formation and a non-singular covariance variance returns matrix for an environment with regime switching.

(A1) Free portfolio formation - The agents can compose any portfolio using the assets in the states of nature. Besides selling an asset in order to fund asset purchases, with a transfer of wealth between times periods, agents can make transfers between states of nature by buying an asset in one state and selling it in another state of nature, providing this state of nature carries out this transition. The wealth of the agents is contingent on the states of nature, in each state the agent has wealth to allocate assets, and the agent invests all of their expected wealth in the assets between the states of nature, so that: $\sum_{i=1}^{N} \omega_{i}(1) P\{1\}+\cdots+\sum_{i=1}^{N} \omega_{i}(s) P\{s\}=1, \omega_{i}(s) \in \mathbb{R}$.

(A2) The covariance variance matrix of returns in each state of nature - $\Omega(s)$ is invertible for each $s \in\{1,2, \ldots, S\}$.

Given the allocation for each regime, the return on the portfolio that occurs in the next period depends on the allocations and returns in each state and on the probability of the regime, where $R^{p}=\omega(1) R(1) P\{1\}+\cdots+\omega(s) R(s) P\{s\}$.

An allocation at the mean variance frontier is the portfolio that minimizes the variance of the portfolio for a given average return. We thus have the following optimization problem: 


$$
\begin{array}{ll}
\min _{\{\omega(1), \cdots, \omega(s)\}} & \sum_{s=1}^{S} \omega^{\prime}(s) \Omega(s) \omega(s) P\{s\} \\
\text { s.t. } & \sum_{s=1}^{S} \omega^{\prime}(s) E(s) P\{s\}=\mu \\
& \sum_{s=1}^{S} \omega^{\prime}(s) \iota P\{s\}=1
\end{array}
$$

whose solution is given by the following proposition.

Proposition 4.1 : For a portfolio at the mean variance frontier with regime switching, the weights of the portfolio for a given mean return on allocation $(\mu)$ are given by:

$$
\omega=\sum_{s=1}^{S} \Omega^{-1}(s)\left[\frac{E(s)(\widetilde{c} \mu-\widetilde{b})+\iota(\widetilde{a}-\widetilde{b} \mu)}{\widetilde{a} \widetilde{c}-\widetilde{b}^{2}}\right] P\{s\}, \forall s \in\{1,2, \ldots, S\}
$$

where $\widetilde{a}=\sum_{s=1}^{S} E^{\prime}(s) \Omega^{-1}(s) E(s) P\{s\} ; \quad \tilde{b}=\sum_{s=1}^{S} E^{\prime}(s) \Omega^{-1}(s) \iota P\{s\}$ and $\widetilde{c}=$ $\sum_{s=1}^{S} \iota^{\prime} \Omega^{-1}(s) \iota P\{s\}$. This way the vector of portfolios contigent on the state at the mean variance frontier with regime switching is expressed by:

$$
\left[\Omega^{-1}(1)\left[\frac{E(1)(\widetilde{c} \mu-\widetilde{b})+\iota(\widetilde{a}-\widetilde{b} \mu)}{\widetilde{a} \widetilde{c}-\widetilde{b}^{2}}\right], \cdots, \Omega^{-1}(s)\left[\frac{E(s)(\widetilde{c} \mu-\tilde{b})+\iota(\widetilde{a}-\tilde{b} \mu)}{\widetilde{a} \widetilde{c}-\widetilde{b}^{2}}\right]\right]
$$

Proof The Lagrangian of the minimization problem is

$$
\begin{aligned}
L(\omega(s), \lambda, \delta)= & \sum_{s=1}^{S} \frac{1}{2} \omega^{\prime}(s) \Omega(s) \omega(s) P\{s\}+\lambda\left[\mu-\sum_{s=1}^{S} \omega^{\prime}(s) E(s) P\{s\}\right]+ \\
& \delta\left[1-\sum_{s=1}^{S} \omega^{\prime}(s) \iota P\{s\}\right]
\end{aligned}
$$

The first order conditions are:

$$
\begin{gathered}
\frac{\partial L}{\partial \omega(s)}=\Omega(s) \omega(s) P\{s\}-\lambda E(s) P\{s\}-\delta \iota P\{s\}=0, \forall s \in\{1,2, \ldots, S\} \\
\frac{\partial L}{\partial \lambda}=\mu-\sum_{s=1}^{S} \omega^{\prime}(s) E(s) P\{s\}=0
\end{gathered}
$$




$$
\frac{\partial L}{\partial \delta}=1-\sum_{s=1}^{S} \omega^{\prime}(s) \iota P\{s\}=0
$$

So

$$
\begin{gathered}
\Omega(s) \omega(s) P\{s\}=\lambda E(s) P\{s\}+\delta \iota P\{s\}, \forall s \in\{1,2, \ldots, S\} \\
\sum_{s=1}^{S} \omega^{\prime}(s) E(s) P\{s\}=\mu \\
\sum_{s=1}^{S} \omega^{\prime}(s) \iota P\{s\}=1
\end{gathered}
$$

From (4)

$$
\omega(s) P\{s\}=\Omega^{-1}(s)[\lambda E(s) P\{s\}+\delta \iota P\{s\}], \forall s \in\{1,2, \ldots, S\}
$$

Multiplying (7) by $E^{\prime}(s)$ for each $s \in\{1,2, \ldots, S\}$, then summing and using (5)

$$
\begin{aligned}
E^{\prime}(s) \omega(s) P\{s\} & =E^{\prime}(s) \Omega^{-1}(s)[\lambda E(s) P\{s\}+\delta \iota P\{s\}], \forall s \in\{1,2, \ldots, S\} \\
\sum_{s=1}^{S} E^{\prime}(s) \omega(s) P\{s\} & =\sum_{s=1}^{S} E^{\prime}(s) \Omega^{-1}(s)[\lambda E(s) P\{s\}+\delta \iota P\{s\}] \\
\mu & =\sum_{s=1}^{S} E^{\prime}(s) \Omega^{-1}(s)[\lambda E(s) P\{s\}+\delta \iota P\{s\}]
\end{aligned}
$$

Multiplying (7) by $\iota^{\prime}$ for each $s \in\{1,2, \ldots, S\}$, then summing and using (6)

$$
\begin{aligned}
\iota^{\prime} \omega(s) P\{s\} & =\iota^{\prime} \Omega^{-1}(s)[\lambda E(s) P\{s\}+\delta \iota P\{s\}], \forall s \in\{1,2, \ldots, S\} \\
\sum_{s=1}^{S} \iota^{\prime} \omega(s) P\{s\} & =\sum_{s=1}^{S} \iota^{\prime} \Omega^{-1}(s)[\lambda E(s) P\{s\}+\delta \iota P\{s\}] \\
1 & =\sum_{s=1}^{S} \iota^{\prime} \Omega^{-1}(s)[\lambda E(s) P\{s\}+\delta \iota P\{s\}]
\end{aligned}
$$


From (8) and (9)

$$
\left[\begin{array}{cc}
\sum_{s=1}^{S} E^{\prime}(s) \Omega^{-1}(s) E(s) P\{s\} & \sum_{s=1}^{S} E^{\prime}(s) \Omega^{-1}(s) \iota P\{s\} \\
\sum_{s=1}^{S} \iota^{\prime} \Omega^{-1}(s) E(s) P\{s\} & \sum_{s=1}^{S} \iota^{\prime} \Omega^{-1}(s) \iota P\{s\}
\end{array}\right]\left[\begin{array}{l}
\lambda \\
\delta
\end{array}\right]=\left[\begin{array}{l}
\mu \\
1
\end{array}\right]
$$

Simplifying the notation

$$
\left[\begin{array}{cc}
\widetilde{a} & \widetilde{b} \\
\widetilde{b} & \widetilde{c}
\end{array}\right]\left[\begin{array}{l}
\lambda \\
\delta
\end{array}\right]=\left[\begin{array}{l}
\mu \\
1
\end{array}\right]
$$

The system solution is:

$$
\begin{gathered}
{\left[\begin{array}{l}
\lambda \\
\delta
\end{array}\right]=\left[\begin{array}{ll}
\widetilde{a} & \widetilde{b} \\
\widetilde{b} & \widetilde{c}
\end{array}\right]^{-1}\left[\begin{array}{l}
\mu \\
1
\end{array}\right]} \\
{\left[\begin{array}{l}
\lambda \\
\delta
\end{array}\right]=\left[\begin{array}{c}
\frac{(\widetilde{c} \mu-\widetilde{b})}{\widetilde{a} \widetilde{c}-\widetilde{b}^{2}} \\
\frac{(\widetilde{a} \mu)}{\widetilde{a} \widetilde{c}-\widetilde{b}^{2}}
\end{array}\right]} \\
\lambda=\frac{(\widetilde{c} \mu-\widetilde{b})}{\widetilde{a} \widetilde{c}-\widetilde{b}^{2}} \\
\delta=\frac{(\widetilde{a}-\widetilde{b} \mu)}{\widetilde{a} \widetilde{c}-\widetilde{b}^{2}}
\end{gathered}
$$

The portfolio over the mean variance frontier with regime switching for a given mean return on the portfolio, is given by the substitution of (12) and (13) into (7), that is:

$$
\begin{aligned}
\omega(s) P\{s\} & =\Omega^{-1}(s)\left[E(s) P\{s\} \frac{(\widetilde{c} \mu-\widetilde{b})}{\widetilde{a} \widetilde{c}-\widetilde{b}^{2}}+\iota P\{s\} \frac{(\widetilde{a}-\widetilde{b} \mu)}{\widetilde{a} \widetilde{c}-\widetilde{b}^{2}}\right] \\
\omega(s) P\{s\} & =\Omega^{-1}(s)\left[\frac{E(s) P\{s\}(\widetilde{c} \mu-\widetilde{b})+\iota P\{s\}(\widetilde{a}-\widetilde{b} \mu)}{\widetilde{a} \widetilde{c}-\widetilde{b}^{2}}\right], \forall s \in\{1, \ldots, S\}
\end{aligned}
$$


Summing and defining $\omega=\sum_{s=1}^{S} \omega(s) P\{s\}$, then using (14)
\[ \omega=\sum_{s=1}^{S} \Omega^{-1}(s)\left[\frac{E(s) P\{s\}(\widetilde{c} \mu-\widetilde{b})+\iota P\{s\}(\widetilde{a}-\widetilde{b} \mu)}{\widetilde{a} \widetilde{c}-\widetilde{b}^{2}}\right] \]

Factoring $P\{s\}$

$$
\omega=\sum_{s=1}^{S} \Omega^{-1}(s)\left[\frac{E(s)(\widetilde{c} \mu-\widetilde{b})+\iota(\widetilde{a}-\widetilde{b} \mu)}{\widetilde{a} \widetilde{c}-\widetilde{b}^{2}}\right] P\{s\}
$$

with portfolios contingent on the states given by:

$$
\omega(s)=\Omega^{-1}(s)\left[\frac{E(s)(\widetilde{c} \mu-\widetilde{b})+\iota(\widetilde{a}-\widetilde{b} \mu)}{\widetilde{a} \widetilde{c}-\widetilde{b}^{2}}\right] \forall s \in\{1,2, \ldots, S\}
$$

with only one state we return to an analytical solution at the mean variance frontier without regime switching ${ }^{2}$.

In the analysis of the first order condition for the expected portfolio conditional on the states, the sum of the first order conditions weighted by the probabilities of the states is proportional to the expected return on the portfolio conditional on the states, $\left[\sum_{s=1}^{S} \Omega(s) \omega(s) P\{s\}\right]=\delta\left[\iota+\sum_{s=1}^{S}\left(\frac{\lambda}{\delta}\right) E(s) P\{s\}\right]$. The assets with the highest expected return will have the greatest allocation and those with the greatest expected variance will have the lowest allocation; however, the highest returns and estimated covariates are in the states that have the lowest probability of occurrence. This attenuates the allocations concentrated in the assets with the highest expected return and lowest risk of the allocations at the mean variance efficient frontier.

From the first order condition for the allocation in each state we have $\Omega(s) \omega(s) P\{s\}=\lambda E(s) P\{s\}+\delta \iota P\{s\}, \forall s \in\{1,2, \ldots, S\}$. Thus, in each state in the allocation of each asset the contribution of the portfolio variance is proportional

2 The case with a single regime corresponds to that in which $P(S=1)=1$ and $P(S=2)=0$. 
to the expected return on the portfolio. Also, the rate of substitution between assets $k$ and $l$ in states $s_{1}$ and $s_{2}$ obeys:

$$
\frac{\Omega\left(s_{1}\right) \omega_{k}\left(s_{1}\right)}{\Omega\left(s_{2}\right) \omega_{l}\left(s_{2}\right)}=\frac{1+\left(\frac{\lambda}{\delta}\right) \delta \mu^{k}\left(s_{1}\right)}{1+\left(\frac{\lambda}{\delta}\right) \delta \mu^{l}\left(s_{2}\right)}
$$

The assets will have greater substitution between states the greater their expected return and the lower their contribution to the relative volatility between the states.

The expected portfolio at the efficient frontier with regime switching is the expected minimum variance portfolio for a given expected return and has second order stochastic dominance over any portfolio that has the same expected return. This result is a consequence of the second order stochastic dominance of the minimum variance portfolio for a given mean return.

Proposition 4.2 : The expected portfolio at the efficient frontier with regime switching has second order stochastic dominance over any expected portfolio with an equal mean return.

Proof The minimum variance portfolio has second order stochastic dominance over any allocation with the same mean return, providing the plan of portfolios contingent on the states is a solution to (4). Where the distribution of the returns have regime switching, the expected portfolio contingent on the states has an expected return and variance in the expected return on the allocation, so that $\omega(s) \forall s \in\{1,2, \ldots, S\}$ is the portfolio contingent on state $s$ at the efficient frontier with regime switching. So the expected portfolio conditional on the states is the only expected portfolio (because of the unicity of the solution) at the efficient frontier with regime switching, being the expected minimum variance portfolio for a given average return, and consequently with second order stochastic dominance over other portfolios with the same mean return.

\section{Empirical Study of Allocations}

Asset allocations can be made based on different models. This section evaluates the comparative performance of allocations based on the regime switching approach with 
other asset allocation models of reference and benchmarks in the literature. Then section 5.1 presents the data used and the procedures adopted to carry out the allocations, and section 5.2 evaluates the performance of the return on the different portfolios. $^{3}$

\subsection{Description of the Data}

In the asset allocation analysis the most liquid stocks that form part of the IBOVESPA and with a large extension of historical data were considered. All the data were extracted from ECONOMÁTICA and the period considered ran from 2003 to 2013. Three criteria were used to include the assets in the sample: assets that form part of the IBOVESPA index in 2013; that are among the 20 assets with the greatest average daily liquidity in the period; and that have transactions on more than $90 \%$ of trading days. This guarantees liquidity and historical data covering 11 years. The assets considered to compose the portfolios are: PETR4, VALE5, ITUB4, BBDC4, VALE3, PETR3, USIM5, GGBR4, BBAS3, CSNA3, ITSA4, CMIG4, CCRO3, BRAP4, PCAR4, ELET6, LAME4, ELET3, GOAU4, and BRKM5. As benchmarks for evaluating the performance of the portfolios, the IBOVESPA index was considered as a reference index for the risk assets, and the CDI as a risk free asset reference.

The behavior of the asset prices and returns are presented in Figures 1 and 2 . The prices are unstable, with strong changes in tendencies in the periods of crisis and global recessionary movements, and the returns move between periods of high and low volatility over time. The subprime crisis in particular caused a heavy fall in prices and considerable volatility in returns on assets.

Table 1 presents the estimation of the model without Markov switching (MVND) and with Markov switching (MS(2)-MVND). All the information criteria indicate that the model with regime switching adjusts better than the normal multivariate distribution without regime switching.

The periods with the greatest volatility in the IBOVESPA coincide with the greatest probability of crisis, calculated by the multivariate model with regime switching (Figure 3).

3 The description of the models and the respective benchmarks are described in appendix. 
Figure 1

Prices of the Selected Risk Assets (Jan/2003 to Dec/2013)

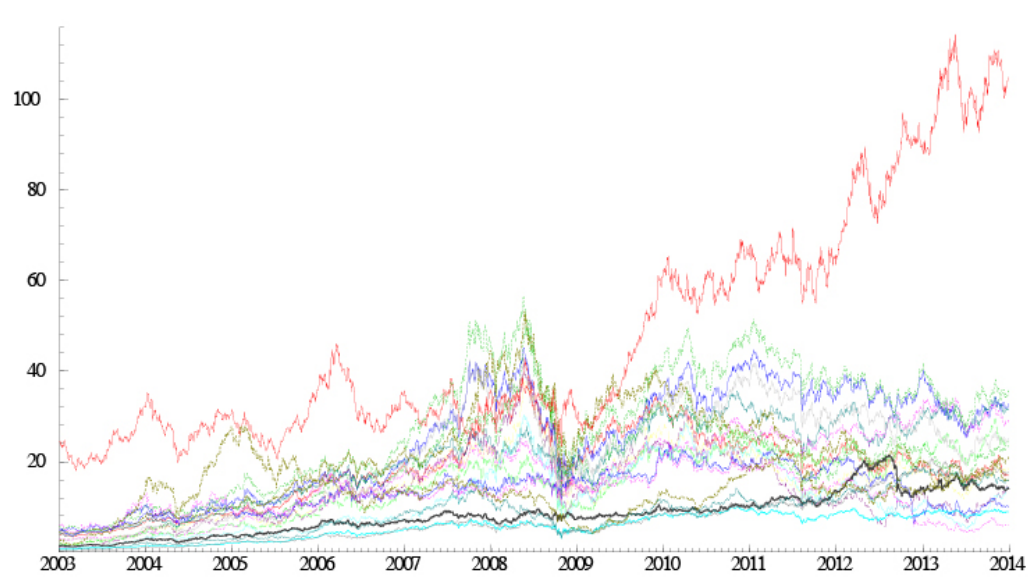

Table 1

Information Criteira: Linear Model with Markovian Regime Switching

\begin{tabular}{ccc}
\hline & MS(2)-MVND & MVND \\
\hline log likelihood & -96272.748 & -99094.196 \\
AIC & 71.417 & 73.329 \\
HQ & 71.781 & 73.510 \\
SC & 72.424 & 73.830 \\
\hline
\end{tabular}

Note: MS(2)-MVND indicates the model with normal multivariate distribution with Markovian size regime switching, with 2 regimes; MVDN denotes the multivariate nomral distribution; (2) The estimation sample was for the period from Jan/2003 to Dec/2003.

In the period from 2003 to 2013, the IBOVESPA was affected by various international crises (Figure 4). Between the first half of 2003 and the first half of 2008 the index rose strongly in valuation. The IBOVESPA valuation movement is interrupted by the subprime crisis between the second half of 2008 and the first half of 2009, when the index reached the lowest point in the dip, and remains in recovery until reaching a level close to 73,000 points in the first half of 2010. Between the second half of 2008 and the first half of 2010 we see strong volatility in IBOVESPA returns. The subprime crisis period characterized a strong global recessionary move- 
Figure 2

Returns on the Selected Assets (Jan/2003 to Dec/2013)

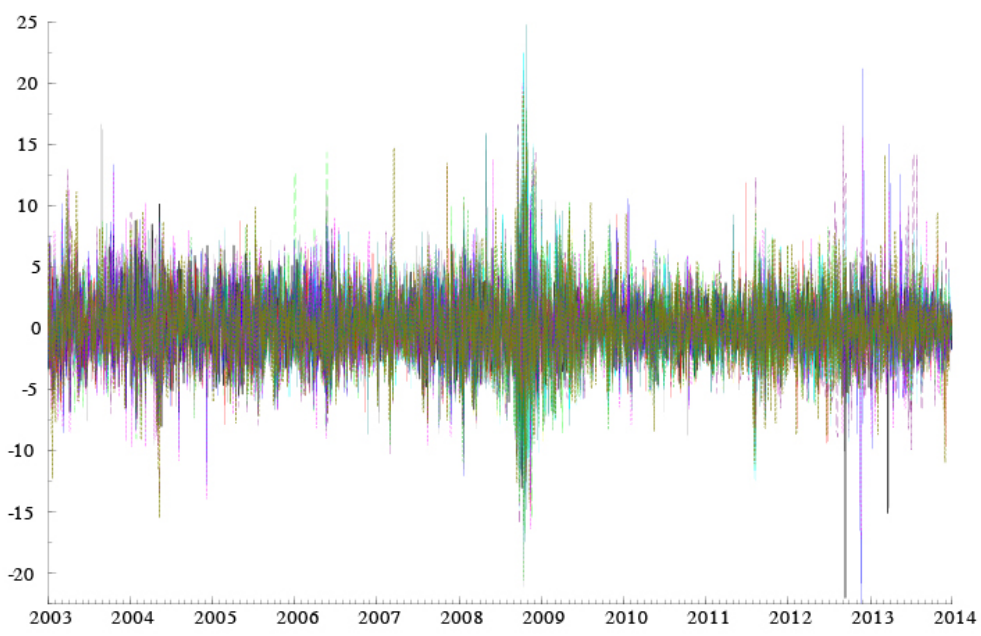

ment with falls in production and unemployment rates comparable with the global crisis of 1929 .

The movement towards growth in the IBOVESPA after the subprime crisis was interrupted by the Greek financial crisis at the start of 2010. Between the end of the second half of 2010 and the first half of 2011 the crisis begins to spread to the Euro Zone, first reaching Ireland and then Portugal, and these movements of uncertainty in the face of a new global crisis caused falls and volatility in the IBOVESPA. In the second half of 2012 we see a new fall in the IBOVESPA with the Euro Zone crisis reaching Spain. The last volatility movement in the IBOVESPA occurs with the financial crisis in Cyprus in the first half of 2013, as the last global recessionary movement of the Euro Zone crisis. During the initial period of the Euro Zone crisis the IBOVESPA is barely volatile, but with the expansion of the crisis to more countries in 2011 we see more volatility and more pronounced market movements.

In the portfolio performance analysis, two evaluation periods are used: the interval between the second half of 2010 and the end of 2013, with an examination in bull and bear markets; and the interval of the second half of 2008, which forms the period of the stress scenario during the subprime crisis. The evaluation period 
Figure 3

Probabilities of Regimes, Multivariate Normal Distribution with Regime Switching (Jan/2003 to Dec/2013).
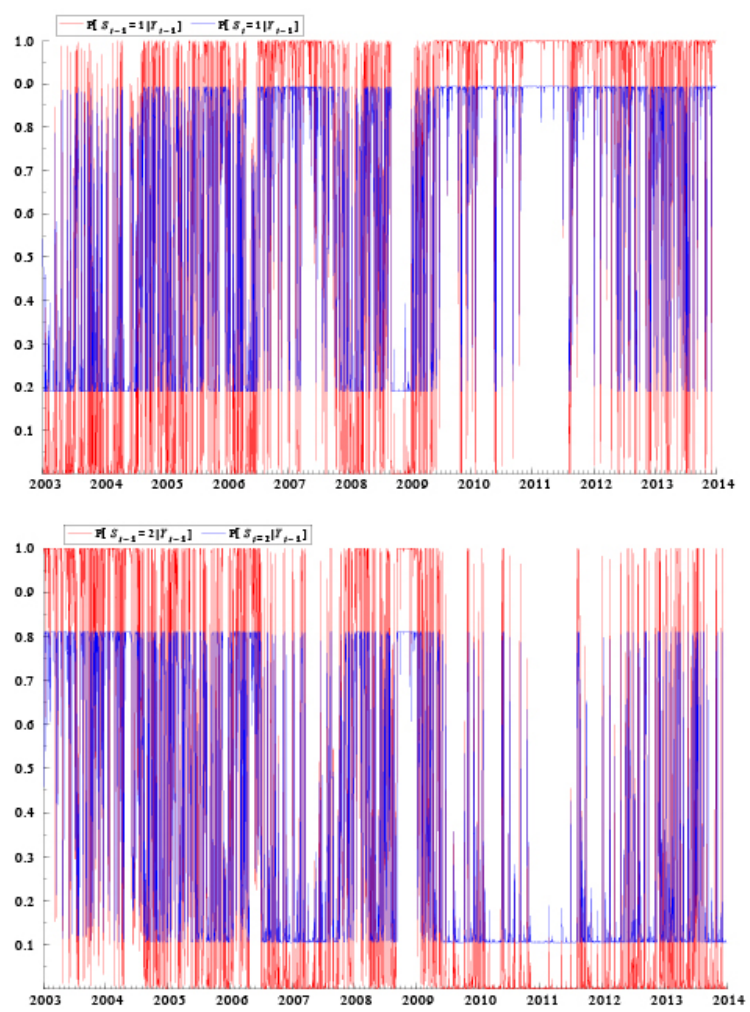

is divided into three moments: (i) a period of low volatility in the market, with bull market in the economy between 1stSem/2010 and 1stSem/2011; (ii) a period of high volatility, with bear market between 1stSem/2011 and 1stSem/2013; (iii) a stress test is also carried out to evaluate the performance of the portfolios between $05 / 2008$ and $11 / 2008$ in a context of extreme volatility between the start of the fall in the IBOVESPA and the minimum quotation of the dip during the subprime crisis.

To make the allocations we use the daily log-returns on a centesimal scale, $r_{t}=$ $100 * \ln \left(P_{t} / P_{t-1}\right)$, where $P_{t}$ is the price at $t$ and $P_{t-1}$ at $t-1$, with a growing window 
Figure 4

IBOVESPA Index and Returns (Jan/2003 to Dec/2013).

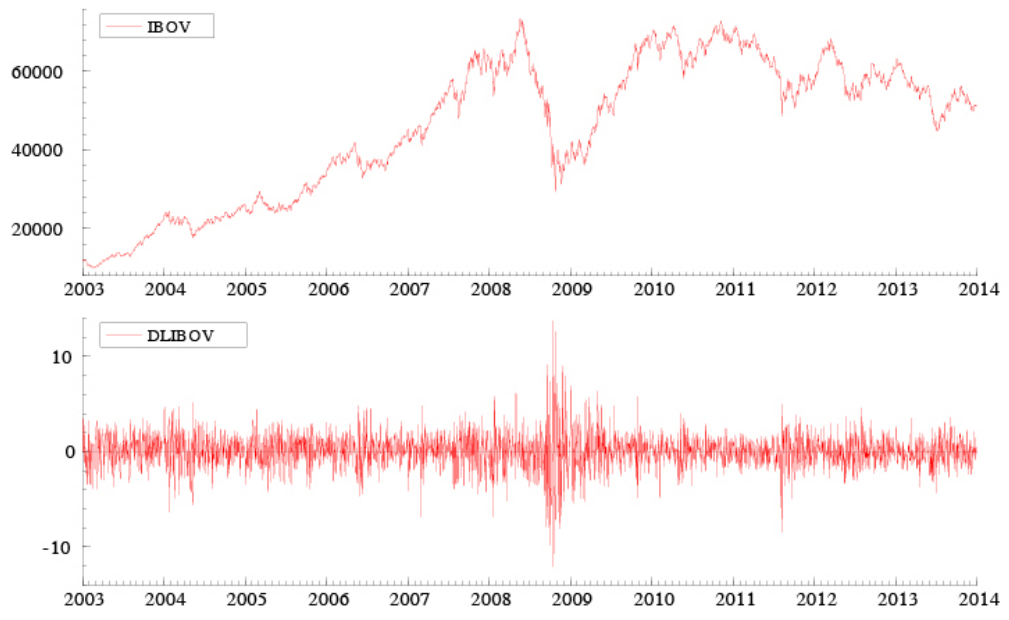

for the estimations with a minimum sample estimation period of 5 years. The allocations are made for the period of one month, with the estimations for the allocation of one month using the sample up to the day immediately before the first day with a recorded quotation for the month of allocation. So, the allocations between 2nd Sem/2010 and 2nd Sem/2013 use a growing window beginning in 2nd Sem/2005. The allocations during the subprime crisis use a sample from 1st Sem/2003. The asset allocations are made using the prediction of risks and returns for the first day after the end of the estimation sample, the last observation of the asset returns combines all the market information, and the asset prices are established starting on the last market observation (Hull (2014) and Shreve (2004)). The mean variance portfolios are defined using their sampling estimates as expected risks and returns. The portfolios with regime switching are executed with the predictions of the joint distribution of the returns with regime switching for the risks and returns for the next day outside the estimation sample.

The Asset Allocation Models with Regime Switching and the others that will be used as benchmarks are presented in appendix. 


\subsection{Comparative Performance of the Portfolios}

Comparing the efficient frontier of the traditional portfolio and the one that uses the model with switching (Figure 5), we can observe that the latter has a lower risk for each level of expected return at the mean variance efficient frontier. Moreover, for efficient frontiers with restricted short selling there are levels of expected returns at the efficient frontier with regime switching that cannot be obtained by the mean variance efficient frontier.

\section{Figure 5}

Efficient Frontier with Regime Switching vs. Mean Variance Efficient Frontier.

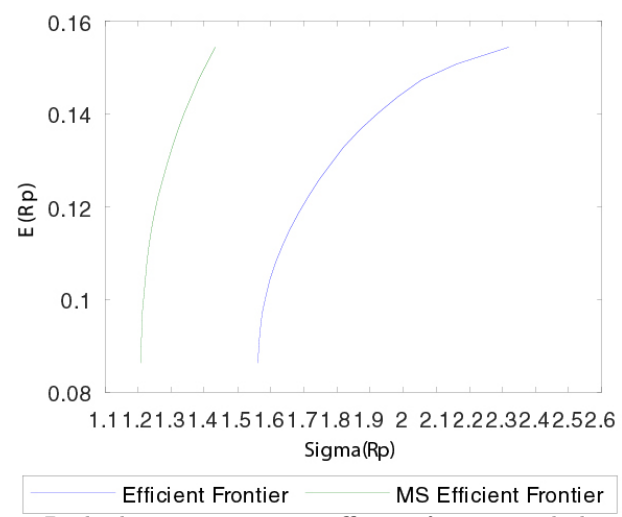

Note: Both the mean variance efficient frontier and the efficient frontier with regime switching were constructed with a restriction on short selling operations, combining the risk and returns pairs for 20 portfolios the global minimum variance portfolio and the maximum mean return between the mean return on portfolio at the mean variance efficient frontier. The efficient frontier with regime switching and mean variance efficient frontier were defined with the estimation sample of the daily returns between Jan/2003 and Dec/2013.

Asset allocations with regime switching and mean variance were carried out for each month. The statistics for the monthly returns on each portfolio are presented in Table 2. For the complete evaluation period (07/2010 - 12/2013), the portfolios with the highest expected return are the ones with regime switching, first the tangent portfolio with conditional regime switching, followed by the tangent portfolio with regime switching. The traditional mean variance portfolio has a less favorable performance in relation to the portfolios with regime switching, with the minimum variance portfolio having the third highest mean return, followed by the tangent 
portfolio. The tangent portfolio with regime switching is the one with the lowest risk out of all the portfolios, followed by the tangent portfolio and then by the global minimum variance portfolio. The higher mean monthly return of the tangent portfolio with conditional regime switching is accompanied by the highest risk out of all of the portfolios based on optimization algorithms.

The heuristic rule of the equally weighted portfolio has the lowest performance in risk and return. The worst performance according to the Sharpe ratio criterion is that of the equally weighted portfolio.

The Sharpe ratio is the main metric for portfolio performance, considering returns penalized by risk. Considering the statistics of the monthly returns on the portfolios using the Sharpe ratio criterion during the whole assessment period, the portfolio with the best performance is the tangent portfolio with regime switching, with an $85 \%$ higher Sharpe ratio than the global minimum variance portfolio. During the low volatility periods the portfolio with the best performance is the tangent portfolio with regime switching, while in the high volatility periods the portfolio with the best performance is the tangent portfolio with conditional regime switching.

In the stress scenario, between the peak of the IBOVESPA and its lowest quotation recorded during the subprime crisis, all the portfolios have negative average returns resulting in a negative Sharpe ratio. All the portfolios perform badly during the stress scenario. With a negative Sharpe ratio we should not invest in risky assets, and the optimal choice is to hold sell positions in risky assets and buy positions in the risk free asset. The short selling restriction maintained in all the portfolios and in all the analysis periods explains the negative returns and loss of the portfolios. 


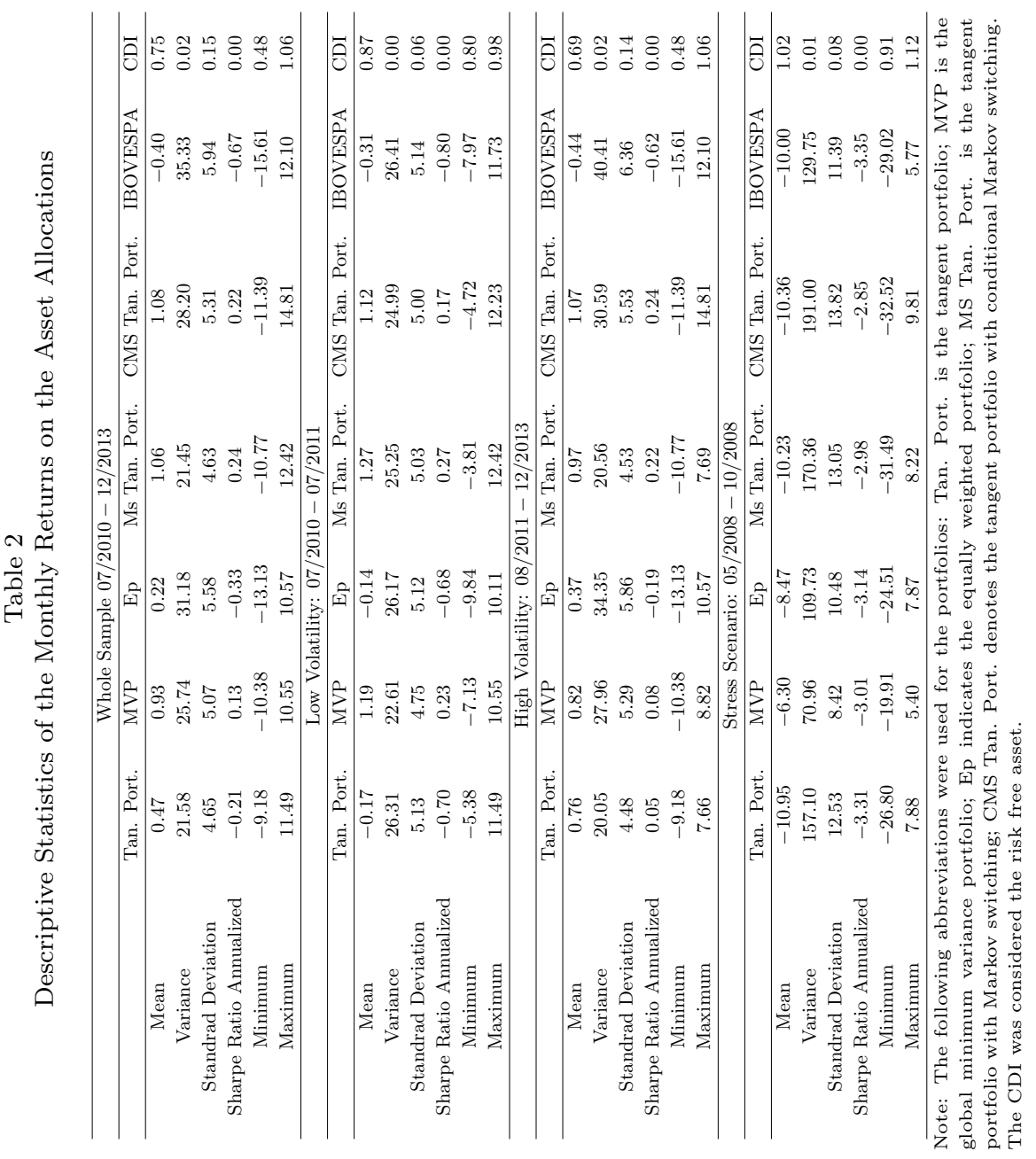


In the stress period the portfolio with the smallest loss and lowest risk is the global minimum variance portfolio. The portfolios with regime switching have higher losses and higher risks. However, the portfolios with regime switching achieve their ex ante objective of minimizing the Sharpe ratio, and they are the ones with the highest Sharpe ratio outside the estimation sample (lowest absolute value between the negative Sharpe ratios), although they are achieved with greater volatility, which results in a reduction in the Sharpe ratio.

We can obtain a greater understanding of the nature of risk diversification for different portfolios by evaluating the characteristics of asset allocations (Table 3). The higher the number of assets in a portfolio, the greater its risk diversification tends to be, with a lower mean allocation in the assets. By definition, the most diversified portfolio is the equally weighted portfolio. Among the optimizationbased portfolios, the most diversified one with the lowest mean allocation is the global minimum variance portfolio. The least diversified portfolio, with the highest mean allocation, is the tangent portfolio. The portfolio with the lowest risk and the lowest standard deviation in monthly returns, however, is the tangent portfolio with regime switching, which combines a low mean allocation, higher only than the mean allocation of the minimum variance portfolio, and maximum allocation in only one asset, in a portfolio reaching $76 \%$ of wealth.

The portfolios using the mean variance approach have risk diversification based on investments in a greater number of assets. On the other hand, the portfolios using the regime switching approach have sequentially adaptive diversification, based both on investments in varied assets and alterations in their portfolios over time in light of alterations in the probabilities of the regimes. The mean variance portfolios are more stable than the portfolios that use the regime switching approach.

Investors are interested in maximizing the return on their portfolios, but they only assume risks that are rewarded by higher returns. From the analysis of the cumulative return on the portfolios (Table 4 and Figure 6), the portfolio with the highest cumulative return and the highest net return on an investment of 100 monetary units on $06 / 30 / 2010$, with a return of approximately $58 \%$, is the tangent portfolio with conditional regime switching, followed by the tangent portfolio with regime switching, with $56 \%$, and the minimum variance portfolio, with $48 \%$. The 
Table 3

Diversification Characteristics of the Portfolios (7/2010-12/2013)

\begin{tabular}{lccccc}
\hline Characteristics & Tan. Port. & MVP & Ep & MS Tan. Port. & CMS Tan. Port. \\
\hline $\begin{array}{l}\text { Average number of } \\
\text { assets per allocation }\end{array}$ & 3.67 & 7.57 & 20 & 7 & 6.69 \\
$\begin{array}{l}\text { Average allocation } \\
\text { between the assets }\end{array}$ & $29.55 \%$ & $13.40 \%$ & $5.00 \%$ & $15.17 \%$ & $15.78 \%$ \\
$\begin{array}{l}\text { with allocation } \\
\text { Maximum allocation }\end{array}$ & $77.06 \%$ & $31.52 \%$ & $5.00 \%$ & $76.22 \%$ & $78.25 \%$ \\
\hline
\end{tabular}

Note: The following abbreviations were used for the portfolios: Tan. Port. is the tangent portfolio; MVP is the global minimum variance potfolio; Ep indicates the equally weighted portfolio; MS Tan. Port. is the tangent portfolio with Markov switching; CMS Tan. Port. denotes the tangent portfolio with conditional Markov switching

mean variance approach portfolio with the highest cumulative return was the global minimum variance portfolio.

In the periods of low volatility, the portfolio with the highest cumulative return was the global minimum variance portfolio, with a return of $25 \%$, around $5 \%$ more than the portfolios with regime switching. In the periods of high volatility, the portfolio with the highest cumulative return was the tangent portfolio with regime switching, with a $32 \%$ return, exceeding the global minimum variance portfolio by more than $10 \%$. During the stress scenario of the subprime crisis, all the portfolios have negative returns, falling together with the IBOVESPA, while the risk free asset maintains the highest return in the period.

The portfolios that tend to maintain a cumulative return that is above the CDI over time are those involving the regime switching approach and the global minimum variance portfolio (Figure 6). During the low volatility periods, the cumulative returns on the portfolios with regime switching are very similar to those of the global minimum variance portfolio. During the periods of high volatility, the returns on the portfolios with regime switching exceed the returns on the minimum variance portfolio.

The volatility of the tangent portfolio with regime switching tends to be lower and more stable than the volatility of the minimum variance portfolio (Figure 7), and the market risks affect the global minimum variance portfolio more over time than the portfolio with regime switching. The tangent portfolio with regime switching has a better risk/return relationship than the other portfolios over time (Figure 6 and Figure 7). 
Table 4

Cumulative Return (\%) on the Portfolios: selected periods

\begin{tabular}{cccccccc}
\hline Periods & Tan. Port. & MVP & Ep & MS Tan. Port. & CMS Tan. Port. & IBOVESPA & CDI \\
\hline $07 / 2010-12 / 2013$ & 21.94 & 48.00 & 9.49 & 56.35 & 57.58 & -15.47 & 36.86 \\
$07 / 2010-06 / 2011$ & 0.87 & 24.91 & 7.23 & 20.30 & 19.29 & 2.41 & 10.87 \\
$07 / 2011-12 / 2013$ & 20.89 & 18.48 & 2.10 & 29.97 & 32.10 & -17.46 & 23.44 \\
$05 / 2008-10 / 2008$ & -48.16 & -31.49 & -39.83 & -45.86 & -46.30 & -45.11 & 6.28 \\
\hline
\end{tabular}

Note: The following abbreviations were used for the portfolios: Tan. Port. is the tangent portfolio; MVP is the global minimum variance potfolio; Ep indicates the equally weighted portfolio; MS Tan. Port. is the tangent portfolio with Markov switching; CMS Tan. Port. denotes the tangent portfolio with conditional Markov switching.

Figure 6

Cumulative Return on the Portfolios (7/2010-12/2013).

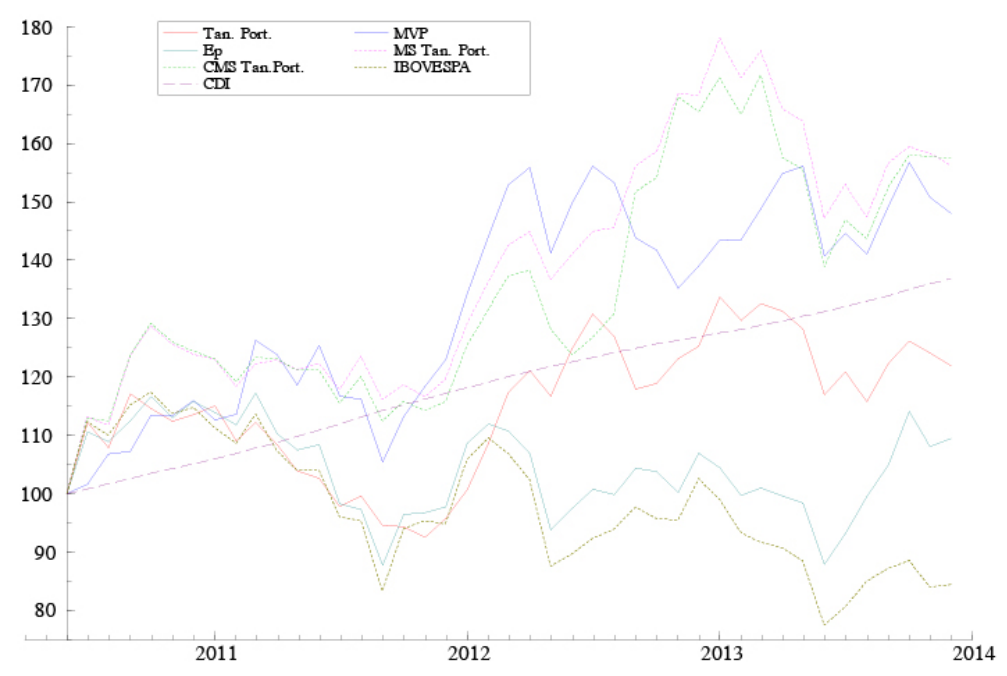

\section{Final Remarks}

Asset allocation has a consolidated portfolio theory; however, the empirical results of asset allocations do not always perform well. In this paper, a theory for asset allocation with regime switching was developed, which analyzes asset allocation by incorporating empirical elements, obtaining better risk and return performance than the traditional approaches.

The joint distribution of returns as a process with regime switching requires a reformulation of the asset allocation problem. In this case the analysis of asset allocation invariant to states becomes a problem with a specification error. In an 


\section{Figure 7}

Volatility of the Portfolio Returns (7/2010-12/2013).

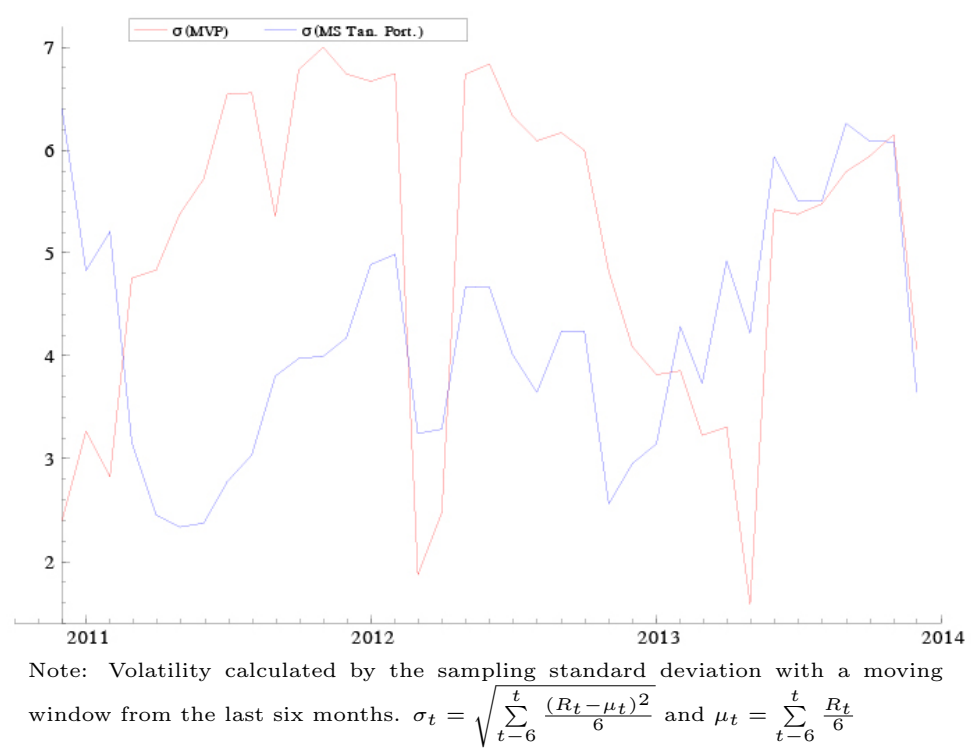

environment with regime switching, the optimal choice of portfolio depends on the probabilities of the states of nature and the agents' optimal portfolio choice set is represented by the efficient frontier with regime switching.

In the empirical evaluation, the asset allocation models with regime switching showed higher performance than the traditional portfolios and benchmarks. The chosen set of efficient portfolios with regime switching, at the efficient frontier with regime switching, presents a risk and return combination for the portfolios with higher returns and lower risk than the mean variance efficient frontier.

In the analysis of the returns on the allocations outside the estimation sample the portfolios with regime switching have higher actual mean returns and lower risks. The Sharpe ratio of the portfolios with regime switching is pronouncedly higher than that of the global minimum variance portfolio and of the tangent portfolio at the mean variance frontier. The portfolios with regime switching enable a better risk-penalized return than the global minimum variance and tangent portfolios. 


\section{References}

Cochrane, J. H. (2005). Asset Pricing. Princeton University Press.

DeMiguel, V., Garlappi, L., \& Uppal, R. (2009). Optimal versus naive diversification: How inefficient is the $1 / \mathrm{n}$ portfolio strategy? The Review of Financial Studies, 22(5):1915-1953.

Franses, P. H. \& van Dijk, D. (2005). Non Linear Time Series Models in Empirical Finance. Cambridge University Press.

Fusai, G. \& Roncoroni, A. (2008). Implementing Models in Quantitative Finance: Methods and Cases. Springer.

Hamilton, J. D. (1989). A new approach to the economic analysis of nonstationary time series and the business cycle. Econometrica, 57(2):357-384.

Hamilton, J. D. (1994). Time Series Analysis. Princeton University Press.

Hamilton, J. D. \& Susmel, R. (1994). Autoregressive conditional heteroskedasticity and changes in regime. Journal of Econometrics, 64(1-2):307-333.

Hull, J. C. (2014). Options Futures and Other Derivatives. Pearson, nineth edition edition.

Jagannathan, R. \& Ma, T. (2003). Risk reduction in large portfolios: Why imposing the wrong constraints helps. The Journal of Finance, 58(4):1651-1683.

Kim, C.-J. \& Nelson, C. R. (1999). State-Space Models with Regime Switching: Classical and Gibbs-Sampling Approaches with Applications. MIT Press.

Krolzig, H.-M. (1997). Markov-Switching Vector Autoregressions: Modelling, Statistical Inference, and Application to Business Cycle Analysis. Springer.

Markowitz, H. (1952). Portfolio selection. The Journal of Finance, 7(1):77-91.

Mas-Colell, A., Whinston, M. D., \& Green, J. R. (1996). Microeconomic Theory. Oxford University Press. 
Michaud, R. O. M. (1989). The markowitz optimization enigma: is 'optimized' optimal? Financial Analysts Journal, 45(1):31-42.

Ross, S. M. (2007). Introduction to Probability Models. Elsiver, ninth edition edition.

Scherer, B. (2002). Portfolio resampling: Review and critique. Financial Analysts Journal, 58(6):98-109.

Shreve, S. (2004). Stochastic Calculus for Finance II. Springer. 


\section{A. Appendix}

\section{Asset Allocation - Optimization Models}

In the study of the asset allocation models with regime switching, traditional portfolio models are compared with portfolios based on the regime switching approach. For all the portfolios, the restriction of non-negativity of weights was introduced, not enabling short selling operations. The description of the models follows.

(a) Global Minimum Variance Portfolio (MVP)

The global minimum variance portfolio is the minimum variance portfolio among all the portfolios at the efficient frontier. The global minimum variance portfolio is the solution to the quadratic programming problem of the minimum variance portfolio among all the portfolios with weights that add up to the unit, given by:

$$
\begin{array}{ll}
\min _{\{\omega\}} & \omega^{\prime} \Omega \omega \\
\text { s.t. } & \omega^{\prime} \iota=1 \\
& \omega_{i} \geq 0, \forall i \in\{1, \cdots, N\}
\end{array}
$$

The global minimum variance portfolio is a portfolio that tends to have the best performance in the comparative studies with other portfolios. The global minimum variance portfolio exceeds the market index with a better risk-penalized return, presenting a higher return and lower risk in relation to the market index (CLARKE, SILVA \& THORLEY, 2006; HAUGEN \& BAKER, 1991). The global minimum variance portfolio in the allocation exercises tends to have a higher Sharpe ratio than the tangent portfolio and other portfolios at the efficient frontier (JAGANNATHAN \& MA, 2003).

(b) Tangent Portfolio (Tan. Port.)

The tangent portfolio is the portfolio that maximizes the Sharpe ratio. The tangent portfolio maximizes the excess return over the risk free asset net of its risk, given by:

$$
\begin{array}{ll}
\max _{\{\omega\}} & \frac{\omega^{\prime} E-R_{f}}{\sqrt{\omega^{\prime} \Omega \omega}} \\
\text { s.t. } & \omega^{\prime} \iota=1 \\
& \omega_{i} \geq 0, \forall i \in\{1, \cdots, N\}
\end{array}
$$

where $E$ is the expected returns vector and $R_{f}$ is the return on the risk free asset. 
The tangent portfolio is the optimal portfolio of risky assets in a portfolio with risky and risk free assets. The tangent portfolio occurs at the tangent between the transformation line and the efficient frontier. Any investor that holds a risk free asset portfolio and risky assets portfolio will hold their risky asset portfolio with the same allocation of risk assets defined by the tangent portfolio, altering only the proportion that they invest in the risky assets portfolio in relation to the risk free asset.

(c) Equally Weighted Portfolio (Ep)

The equally weighted portfolio allocates its income equally between all the risk assets available for allocation, given by: $\omega_{i}=\frac{1}{N}, i \in\{1, \cdots, N\}$. The equally weighted portfolio is an intuitive portfolio for risk diversification.

De Miguel, Garlappi, \& Uppal (2009) evaluate the performance of different portfolios based on the mean variance approach compared with the equally weighted portfolio, considering allocations over sets of market indices, and they find that the equally weighted portfolio has a greater performance than the other portfolios in the various assessment metrics. Jacobs, Muller, \& Weber (2013) compare mean variance approach portfolios with heuristic rules over sectorial and international indices and find that the equally weighted portfolio offers similar diversification gains to the optimization-based portfolios.

(d) Tangent Portfolio with Markov Switching (Tan. Port. MS)

The tangent portfolio with Markovian regime switching is the conditional expected portfolio at the states that maximizes the Sharpe ratio and allocates expected wealth between the portfolios of each state. The tangent portfolio with regime switching maximizes the ratio of the expected excess return divided by the expected standard deviation of the portfolio. In an economy with $s \in S$ possible states, the tangent portfolio with regime switching is given by $\omega=\sum_{s=1}^{S} \omega(s) P\{s\}$, where $\omega(s) s \in\{1, \cdots, S\}$ is the solution to:

$$
\begin{array}{ll}
\max _{\{\omega(1), \cdots \omega(S)\}} & \frac{\sum_{s=1}^{S} \omega^{\prime}(s) E(s) P\{s\}-R_{f}}{\sqrt{\sum_{s=1}^{S} \omega^{\prime}(s) \Omega(s) \omega(s) P\{s\}}} \\
\text { s.t. } & \sum_{s=1}^{S} \omega^{\prime}(s) \iota P\{s\}=1 \\
& \omega_{i}(s) \geq 0, \forall i \in\{1, \cdots, N\} \text { and } s \in\{1, \cdots, S\}
\end{array}
$$


The tangent portfolio with regime switching lies over the efficient frontier with regime switching. The tangent portfolio with regime switching is the optimal choice of expected portfolio of risk assets conditional on the states in a risk free asset portfolio and risky assets portfolio.

(e) Tangent Portfolio with Conditional Markov Switching (Tan. Port. CMS)

The tangent portfolio with conditional Markovian regime switching is a portfolio that maximizes the Sharpe ratio conditional on the states, distributing all the agent's wealth in each state of the economy into the portfolio attributed to the same state, expressed by $\omega=\sum_{s=1}^{S} \omega(s) P\{s\}$, where $\omega(s) s \in\{1, \cdots, S\}$ is the solution to following non-linear programming problem:

$$
\begin{array}{ll}
\max _{\{\omega(s)\}} & \frac{\omega^{\prime}(s) E(s)-R_{f}}{\sqrt{\omega^{\prime}(s) \Omega(s) \omega(s)}} \\
\text { s.t. } & \omega^{\prime}(s) \iota=1 \\
& \omega_{i}(s) \geq 0, \forall i \in\{1, \cdots, N\} \text { and } s \in\{1, \cdots, S\}
\end{array}
$$

The tangent portfolio with conditional regime switching is a direct application of the allocation approach conditional on states. In each state we have an efficient frontier and the tangent portfolio with conditional regime switching is the tangent portfolio at the efficient frontier in each state weighted by the probability of the state.

In this study I consider all the portfolios with short-selling restrictions. The no short-selling restriction means the portfolios are obtained through optimization programs, while the portfolios enabling short-selling have an exact analytical solution. Short-selling operations are difficult and costly to operationalize in practice and constitute a restriction that is sensitive to the agents in the financial market.

The allocations based on the traditional mean variance approach are plug-in portfolios, inserting the estimated sampling moments into the portfolio. One of the main problems of asset allocation using the portfolios in the plug-in approach is that the portfolio optimization considers that the estimated moments are the populational parameters, while the sampling moments are subject to sampling errors. The error in the sampling estimates frequently results in low performance of the plug-in portfolios 
Portfolio theory attempts to overcome the sampling errors and low performance of plug-in portfolios by improving the quality of the sampling estimators with specifications that minimize the sampling errors, such as portfolios with robust estimators or shrinkage estimation. Besides improving the quality of the estimates used in the portfolios, the regime switching approach for asset allocation redefines the specification of the portfolio optimization problem, considering changes in the data generating process during asset allocation and the effect of the uncertainty conditional on the states on the risks and returns. 